\title{
Teto metabólico como indicador de capacidade de tolerância ao esforço físico: importância da capacidade oxidativa de reserva
}

CDD. 20.ed. 796.027

Benedito PEREIRA*

*Escola de Educação Física e Esporte, Universidade de São

http://dx.doi.org/10.1590/1807-55092014000400683

Paulo.

\section{Resumo}

Comparados com outras espécies animais, a capacidade para a resistência de seres humanos é notável. A tese dos fatores limitantes e determinantes da resistência considera variáveis como consumo máximo de oxigênio $\left(\mathrm{VO}_{2 \max }\right)$, limiares metabólicos e economia de movimento as mais importantes para a efetivação de exercícios físicos de longa duração. Como o oxigênio $\left(\mathrm{O}_{2}\right)$ e produtos do metabolismo de substratos (glicose e ácidos graxos) são utilizados no interior de mitocôndrias do tecido muscular para obtenção de energia por processos oxidativos durante esse tipo de exercício físico, esta é outra variável importante a ser considerada. 0 objetivo deste texto é demonstrar que a incidência de fadiga em exercícios físicos prolongados intensos pode estar relacionada com modificações negativas ocorridas no potencial oxidativo mitocondrial. A funcionalidade mitocondrial encontra-se situada entre limites extremos que correspondem aos estados 4 (repouso) e $3\left(\mathrm{VO}_{2 \max }\right)$. A tese desse texto é a de que quando essa variável aproxima-se do estado 3 durante o exercício físico prolongado intenso, o organismo esgota suas possibilidades de produção de energia pela fosforilação oxidativa, com consequente modificações nos valores de limiares metabólicos e no percentual do $\mathrm{VO}_{2 \max }$ utilizado durante o esforço físico. Portanto, mitocôndrias funcionam como um termostato bioenergético celular durante situações como as que envolvem o exercício físico intenso prolongado.

Palavras-chave: Fadiga; Metabolismo; Mitocôndria; Resistência.

\section{Introdução}

O principal objetivo deste texto é demonstrar que a capacidade oxidativa mitocondrial é um importante fator a ser considerado na caracterização da resistência como "capacidade de tolerância ao esforço físico prolongado". As mitocôndrias são importantes para a resistência porque sáo o centro de produção bioenergética oxidativa celular ${ }^{1}$. Portanto, pode-se hipotetizar que a produçáo de trifosfato de adenosina (ATP) e a eficiência desse processo sejam determinantes bioenergéticos do desempenho físicoesportivo (DFE) em atividades físicas prolongadas. Por exemplo, foi demonstrado recentemente que a capacidade oxidativa mitocondrial (fosforilação oxidativa e transporte de elétrons) do músculo esquelético é a variável que integra funcionalmente todas as três mais utilizadas para predizer o DFE de resistência $\left(\mathrm{VO}_{2 \max }\right.$, limiar de lactato e eficiência de movimento). Em função disso, a mesma foi considerada a variável mais importante na predição do desempenho de atletas altamente treinados em atividades de longa duraçãó ${ }^{2}$.

Mitocôndrias respondem de forma quantitativa e qualitativa ao treinamento físico-esportivo (TFE) com exercícios físicos de longa duração ${ }^{3}$. No primeiro caso, ocorre aumento no volume mitocondrial do tecido, enquanto no segundo, somente na atividade de enzimas e processos de transporte e cinética de reaçôes químicas. Além disso, é possível a síntese de proteína a partir de mRNA pré-existente. Entretanto, essas modificaçôes não são confinadas à musculatura esquelética, mas também em outros tecidos metabolicamente ativos como fígado, tecido adiposo e rins ${ }^{4}$. As variáveis volume e duração são mais importantes para a síntese de mitocôndrias, enquanto que sua qualidade funcional depende mais da intensidade.

Como a capacidade oxidativa muscular encontrase em excesso na musculatura esquelética de seres humanos com relação a que é necessária à realização 
de exercícios físicos prolongados, a funcionalidade mitocondrial é considerada mais importante na determinação do DFE neste tipo de atividade física ${ }^{5}$. Isso significa que atletas especialistas em exercícios de longa duração devem priorizar a alta intensidade em seus TFEs. Por outro lado, o destreinamento e o envelhecimento promovem queda na quantidade e funcionalidade mitocondrial. Portanto, nestes casos, o volume e a duraçáo devem ser enfatizados no treinamento ${ }^{3}$.

Em termos funcionais, quando a demanda bioenergética durante o exercício físico é mantida abaixo da "máxima potência oxidativa celular", considera-se que um teto metabólico ("metabolic ceiling") mitocondrial foi estabelecido, sendo possível manter o esforço físico por longo tempo nesse valor de taxa metabólica ${ }^{6}$. Piersma ${ }^{6}$ encontrou valores de teto metabólico entre 30-35 MJ.dia ${ }^{-1}$ para humanos exploradores dos polos da Terra, no "Tour de France" e na multi-maratona no Sahara. Foi verificado que nossa capacidade de sustentação do teto metabólico é bastante limitada, porque quanto maior for o tempo de sustentação de determinado valor de intensidade de esforço físico, menor se torna a quantidade de energia disponibilizada para tanto, atingindo progressivamente um valor que é poucas vezes superior à taxa metabólica basal. Em outras palavras, a potência desenvolvida por animais se exercitando é uma função negativa do tempo de duração da atividade física ${ }^{6}$.

O mecanismo explicativo para a perda progressiva da capacidade de sustentar o esforço físico por longo tempo pode estar relacionado com queda na eficiência bioenergética durante esse tipo de atividade física. Esta hipótese é baseada no simples fato de que a contraçáo muscular depende de ATP e que a eficiência na sua produção mitocondrial é reduzida progressivamente no exercício físico de longa duração ${ }^{7}$. Os dois principais argumentos favoráveis a essa sugestấo são: 1 . O organismo não se encontra totalmente em estado de equilíbrio homeostático durante o esforço físico realizado no teto metabólico e; 2 . As leis termodinâmicas e bioenergéticas consideram que a eficiência é inversamente proporcional à duraçâo e intensidade das transformaçôes bioenergéticas ocorridas no organismo durante o esforço físico ${ }^{8}$. Portanto, aumentar a potência metabólica para sustentar o teto metabólico resulta em elevação no desperdício bioenergético durante o exercício físico prolongado ${ }^{6}$.

\section{Escopo aeróbio fatorial e absoluto}

O valor do teto metabólico correspondente ao ritmo de corrida estabelecido pelo atleta depende do seu escopo aeróbio. Existem dois tipos de escopo aeróbio, que são denominados por "escopo aeróbio fatorial" (EAF) e "escopo aeróbio absoluto". O primeiro é a razão entre a máxima taxa metabólica possível $\left(\mathrm{VO}_{2 \max }\right)$ e o metabolismo basal, enquanto que o segundo, a diferença aritmética entre $\operatorname{ambos}^{10}$. Grande escopo aeróbio propicia que a velocidade de movimento durante a corrida possa ser sustentada sem recorrer de maneira significativa a fontes anaeróbias (não-oxidativas) suplementares $^{11}$. Portanto, quanto maior o escopo aeróbio do organismo, maiores são as possibilidades de que possa sustentar altos valores de teto metabólico durante a corrida prolongada sem incidência de fadiga.

O escopo aeróbio não possui unidade definida, sendo da ordem de seis a 10 para espécies animais sedentárias e de 10 a 60 para espécies consideradas ativas. O EAF pode variar em até 12 vezes em indivíduos não-treinados, em torno de 16 a 21 vezes respectivamente para jogadores de futebol e ciclistas e, em mais de 24 vezes em corredores de longas distâncias? ${ }^{9}$. Portanto, dois indivíduos correndo a maratona mantendo a mesma intensidade de esforço físico podem utilizar, para tanto, diferentes valores de teto metabólico. Como consequência desse fato, aquele que mantêm o menor valor de teto metabólico para a mesma intensidade relativa de esforço é frequentemente considerado o que possui a maior "economia de movimento"12.

O escopo aeróbio também pode ser definido como a fração do fluxo de energia e potência metabólica disponível após os custos da manutenção basal terem sido atendidos. Portanto, se o custo absoluto da manutenção basal for elevado, o escopo aeróbio será reduzido. Por exemplo, quando as variáveis ambientais desviam-se da normalidade ou do valor ótimo para o organismo, o escopo aeróbio diminui devido ao aumento nos custos da manutençáo basal. Isso resulta em limitaçốes ao consumo de substratos e distribuição sistêmica de $\mathrm{O}_{2}$, produção de ATP, etc.

A queda no escopo aeróbio devido à temperatura elevada no meio ambiente é provocada pela capacidade limitada do sistema circulatório e respiratório em atender as necessidades do tecido muscular durante o exercício físico prolongado. O frio, por outro lado, 
pode reduzir essa variável como resultado da baixa capacidade mitocondrial para síntese de ATP. A altitude elevada reduz a $\mathrm{pO}_{2}$ e diminui a disponibilidade do $\mathrm{O}_{2}$ para a fosforilação oxidativa, assim como o teor mitocondrial do tecido muscular. Portanto, a magnitude do valor do escopo aeróbio impóe limites à tolerância à temperatura, valores de $\mathrm{O}_{2}$, poluição, intensidade sustentada de esforço físico, etc. ${ }^{13}$.

Em virtude de os experimentos envolvendo avaliações do DFE serem realizados em condiçóes laboratorias controladas, como no caso daquelas consideradas preditoras de desempenho, modificaçóes intrínsecas ou extrínsecas ao organismo por tempo suficiente podem torná-las irreprodutíveis em condições naturais. Outro problema envolve a não mensuração dos valores da taxa metabólica basal previamente ao $\mathrm{VO}_{2 \max }$, significando que esses experimentos são frequentemente realizados sem se conhecer o escopo aeróbio de sedentários e de atletas.

\section{Capacidade oxidativa de reserva}

Teto metabólico e escopo aeróbio são relacionados por alguns autores com o conceito de "capacidade respiratória de reserva" ("spare respiratory capacity" ou "reserve respiratory capacity") ${ }^{1}$. Capacidade respiratória de reserva foi definida como a diferença entre o ATP produzido pela fosforilação oxidativa para manutenção do metabolismo basal e a sua máxima produção pela atividade respiratória mitocondrial. $\mathrm{O}$ valor dessa reserva é definido pela inter-relação entre reguladores de curto (variação na razão $\mathrm{ADP} / \mathrm{ATP}$ ) e de longo prazo (modificaçôes na síntese enzimática e proteínas mitocondriais) da fosforilação oxidativa. Como essa definição diz respeito à funcionalidade do metabolismo oxidativo de substratos, aqui será conceituada como "capacidade oxidativa de reserva". Por exemplo, foi demonstrado que cinco a 10 dias de exercícios físicos de resistência já são suficientes para modificar reguladores de curto prazo da fosforilaçáo oxidativa e elevar a produção intracelular de fatores ativadores da transcrição ${ }^{14}$. Como será descrito neste trabalho, o mesmo foi verificado para a produção tecidual de mitocôndrias com o TFE por longo tempo utilizando exercícios físicos de longa duração.

Em condições basais de atividade metabólica oxidativa, as mitocôndrias encontram-se no "estado 4". A máxima atividade do sistema de transporte de elétrons equivale ao "estado 3". No estado mitocondrial 4, mesmo na presença de substratos oxidáveis, a mitocôndria consome pouco $\mathrm{O}_{2}$ em baixas concentraçôes de ADP e, no estado mitocondrial 3, na presença de $\mathrm{ADP}$, a respiração torna-se rápida devido à grande demanda por ATP. Portanto, no estado mitocondrial 4, essas organelas encontram-se com alto potencial redox $\left(\mathrm{NADH} / \mathrm{NAD}^{+}\right)$e de membrana $(\triangle \Psi)$ devido à grande oferta de substratos com baixa demanda por energia. O oposto se aplica ao estado 3. Portanto, modificações na demanda bioenergética celular promovem oscilaçôes entre esses dois estados e interfere grandemente na atividade metabólica, com seu valor situando-se normalmente em 3,6. Em funçáo disso, pode-se dizer que mitocôndrias funcionam como um termostato bioenergético celular.

A magnitude do valor da reserva oxidativa é critica para a manutenção da funcionalidade celular durante condiçóes de estresse agudo e crônico, porque quando o estado respiratório mitocondrial é desviado para próximo do estado 3, sua capacidade é esgotada. Foi descrito que em condiçôes em que o valor dessa reserva é ultrapassado pode resultar em morte celular e perda de funcionalidade biológica. Esse efeito foi observado em doenças cardíacas, neurodegenerativas e morte do tecido muscular liso ${ }^{1}$. O envelhecimento, ao afetar a funcionalidade mitocondrial e o controle da fosforilação oxidativa, pode reduzir os seus valo$\mathrm{res}^{15}$. De fato, o $\mathrm{VO}_{2 \max }$ declina com o envelhecimento, mesmo se forem feitas correçóes no valor obtido devido a perda de massa corporal magra.

Outro dado importante, relativo ao envelhecimento, é a queda constatada na velocidade máxima de produção de ATP pelo tecido muscular. O mesmo também se aplica a essa variável na condição basal, porque há queda no estado mitocondrial 4 no músculo esquelético de animais envelhecidos ${ }^{16}$. As próprias condiçôes ambientais desfavoráveis, como descritas antes, alteram negativamente essa reserva. Problemas resultantes do esgotamento da reserva oxidativa podem ser devido à ocorrência de queda na eficiência bioenergética celular devido ao alto valor de potência metabólica desencadeado no organismo para atender a alta demanda celular por energia durante a corrida, como já afirmado antes ${ }^{17}$.

Como o exercício físico intenso aproxima o metabolismo basal do estado 3 e esgota a reserva oxidativa do organismo, fadiga pode estar relacionada 
com proteção do organismo em exercício físico intenso através de modificaçóes ocorridas no potencial bioenergético celular ${ }^{18}$. Ou seja, fadiga, neste caso, é uma forma de prevenção do organismo contra a possibilidade de que a reserva oxidativa seja completamente ultrapassada.

\section{Capacidade oxidativa de reserva no repouso e no exercício}

Existem no repouso duas situaçôes importantes que podem acarretar em desperdício bioenergético com possíveis modificaçóes na reserva oxidativa mitocondrial, a saber: 1 . Na condição de existência de balanço na demanda e oferta de ATP e oferecimento de altas concentraçóes de substratos para sua síntese e; 2. Maior oferta de substratos com queda na demanda bioenergética imediatamente após o exercício físico. $\mathrm{O}$ excesso de nutrientes na ausência de demanda elevada por ATP representa uma situação em que as necessidades por energia são baixas comparadas com a que se encontra presente em substratos ${ }^{19}$. Esse é o caso, por exemplo, da dieta de supercompensação praticada com a intenção de ampliar os estoques intracelulares de glicogênio muscular e hepático.

Foi demonstrado que após o exercício físico o ciclo de Krebs (TCA) encontra-se com atividade elevada (vTCA), abastecendo a cadeia respiratória intensamente com poder redutor, mas com a síntese de ATP inalterada nessa situação em indivíduos treinados comparados com náo-treinados submetidos ao mesmo tipo de esforço físico. Verificaram ainda que a razão vATP/vTCA (indicador de acoplamento mitocondrial) está significativamente reduzida em mitocôndrias de indivíduos treinados comparados aos sedentários no repouso após a realização do esforço físico $^{20}$. Ou seja, o poder redutor elevado intramitocondrialmente, devido ao exercício físico, não é totalmente aproveitado na síntese de ATP por indivíduos treinados imediatamente após o seu término.

Estes exemplos servem para justificar a hipótese de que existem situaçóes em que animais vivenciam limitações fisiológicas, metabólicas e bioenergéticas intrínsecas que ultrapassam suas necessidades de consumo de substratos, sendo sugerido que restriçóes ocorridas em seus desempenhos podem ser por causa de suas incapacidades de utilizar a energia ou fontes disponíveis desses substratos. Esse fenômeno ocorre porque mitocôndrias apresentam, nesta condição, aumento no componente relacionado com ineficiência, que foi denominado por "consumo mitocondrial de $\mathrm{O}_{2}$ controlado por condutância basal de prótons", com a possibilidade de participação de proteínas desacopladoras de membranas denominadas por UCPs ("uncoupler proteins") e outros processos em investigação ${ }^{19}$.

O aumento no fluxo de elétrons na cadeia respiratória devido à grande oferta de poder redutor resulta em elevação no potencial de membrana mitocondrial, com elevação paralela no fluxo de prótons sem que a energia do gradiente seja utilizada na síntese de ATP (neste caso, o potencial de membrana torna-se mais negativo). Foi demonstrado que o potencial de membrana maior ou igual a $-140 \mathrm{mV}$ resulta em desacoplamento da fosforilação oxidativa ${ }^{21}$. Ou seja, parte da energia do gradiente de prótons é dissipada na forma de calor. Os limites fisiológicos para o potencial de membrana mitocondrial encontram-se entre -80 (estado 3) e $-170 \mathrm{mV}$ (estado 4). Pode-se dizer, desta forma, que os extremos desses valores representam a capacidade oxidativa de reserva, que é protegida pelas células e pela própria mitocôndria através de modificações na potência metabólica e no mecanismo de desperdício bioenergético $^{21}$. Isso significa que o mecanismo de desperdício é induzido em mitocôndrias tanto na condição de exercício físico intenso como no repouso.

No caso de sedentários, esse mecanismo pode ser ativado quando a taxa metabólica basal é mantida com decréscimo no teor mitocondrial tecidual. Para manter a taxa metabólica basal com menos mitocôndria tecidual é necessário que ocorra elevação na atividade de suas unidades individuais. Nesta condição, constata-se redução na magnitude da reserva oxidativa mitocondrial porque poucas mitocôndrias consomem $\mathrm{O}_{2}$ mais intensamente em músculos de sedentários comparativamente à maior quantidade de mitocôndrias presentes em músculos de indivíduos treinados ${ }^{22}$. De fato, foi verificado que o fluxo de $\mathrm{O}_{2}$ por ATP produzido encontra-se duplicado em sedentários comparados com músculos de indivíduos ativos ${ }^{17}$.

Neste estudo, foi demonstrado por Conley et al. ${ }^{17}$ que a capacidade funcional dos sistemas de transporte de elétrons encontrava-se normal, mas o conteúdo em sistemas de transporte de elétrons e a máxima produção de ATP apresentavam-se 60\% abaixo do valor obtido de mitocôndrias de músculos ativos. Isso significa que o menor volume mitocondrial tecidual de sedentários possui potência metabólica 
elevada para atender as necessidades bioenergéticas do organismo; resultando, como consequência, em maior desperdício bioenergético comparativamente ao tecido com maior volume mitocondrial. Portanto, mitocôndrias teciduais de sedentários encontramse significativamente mais desacopladas "in vivo", confirmando a presença de ineficiência mitocondrial com manutenção de estabilidade no metabolismo celular, tanto em altas como em baixas exigências bioenergéticas ${ }^{17}$. Ressaltando que altas temperaturas teciduais resultantes desse processo tornam a eficiência bioenergética e metabólica ainda menor ${ }^{23}$.

A principal modificação decorrente do TFE de resistência com possibilidade de ampliaçáo da reserva oxidativa celular relaciona-se com o aumento do número de mitocôndrias teciduais. Com relação à eficiência bioenergética mitocondrial, por motivos desconhecidos, a razão $\mathrm{P} / \mathrm{O}$ (ATP produzido por $1 / 2 \mathrm{O}_{2}$ consumido) permanece igual ou diminuída após o TFE. Inclusive, levando-se em conta o organismo como um todo, nenhuma diferença significativa parece existir nesta variável entre atletas de alto nível e indivíduos sedentários ${ }^{17}$.

Como indivíduos treinados neste tipo de exercício físico possuem a mesma eficiência bioenergética mitocondrial que a de indivíduos sedentários, o principal efeito positivo do maior número de mitocôndrias teciduais na proteçẫo da capacidade oxidativa de reserva decorre do seu efeito redutor na potência metabólica durante o exercício físico, sem diminuir a oferta de ATP e o DFE. Ou seja, mitocôndrias individuais trabalham menos intensamente com a mesma eficiência na produção de ATP quando o seu número encontra-se aumentado ${ }^{24}$. De certa forma, por razōes bioenergéticas e termodinâmicas, pode-se dizer que o menor fluxo de elétrons pela cadeia respiratória em maior número pode favorecer a eficiência bioenergética $^{25}$. Ou seja, é possível que o TFE de resistência induza no músculo esquelético não somente mudanças quantitativas mitocondriais, mas também mudanças na sua qualidade funcional. Essa possibilidade foi parcialmente demonstrada recentemente ${ }^{2}$.

\section{Considerações finais}

Os estudos previamente revisados indicam que os fatores que impóem restriçôes à manutenção de altos valores de potência metabólica quando a demanda bioenergética encontra-se elevada podem estar relacionados com mecanismos de controle da funcionalidade oxidativa mitocondrial. Mitocôndrias
O nitrato consumido na dieta é uma possibilidade que se encontra sob investigação quanto ao seu efeito positivo na eficiência bioenergética. $\mathrm{O}$ nitrato suplementado na dieta demonstra impacto direto na funcionalidade mitocondrial, reduzindo o vazamento de prótons desta organela ${ }^{26}$. No caso do exercício físico e TFE, foi demonstrado que quando a eficiência bioenergética é ampliada através do consumo de nitrato inorgânico, observa-se queda acentuada nos valores do $\mathrm{VO}_{2 \max }$ sem prejudicar o $\mathrm{DFE}^{27}$. Foi sugerido que o treinamento pode induzir aumento corporal na concentração de nitrato de forma natural. Esse fato foi parcialmente comprovado em moradores de grandes altitudes (Tibetanos).

Nativos desse grupo de humanos demonstram baixo consumo de $\mathrm{O}_{2}$ no exercício submáximo, alta eficiência grosseira na locomoçáo, baixo valor de $\mathrm{VO}_{2 \max }$ e 10 vezes mais nitrato na circulação sanguínea comparado com habitantes de baixas altitudes ${ }^{28}$. $\mathrm{O}$ nitrato encontra-se presente, por exemplo, em alimentos industrializados como o salame, salsicha ou outros produtos em conserva ("cured meat"). Portanto, o aumento no volume mitocondrial muscular ou o aumento na sua eficiência propicia que cada uma dessas mitocôndrias apresente atividade próxima ou correspondente ao estado mitocondrial 4.

Estudos recentes demonstraram em ratos que o desacoplamento mitocondrial induzido quimicamente com dinitrofenol resulta: 1 . Em aumento no consumo basal de $\mathrm{O}_{2}$ e reduçáo na velocidade de ganho no peso corporal; 2. Em modificaçóes positivas na quantidade de mitocôndrias teciduais como compensaçáo devido à queda em sua funcionalidade e; 3. Em queda na velocidade máxima durante a corrida, com aumento nos valores do $\mathrm{VO}_{2 \max }$. Em conjunto, esses dados demonstram a importância das propriedades funcionais de mitocôndrias (acoplamento mitocondrial) em adiçáo ao aumento em sua quantidade com o TFE de resistência para a determinação da capacidade máxima de desempenho físico e $\mathrm{VO}_{2 \max }{ }^{29}$.

são, desta forma, bastante flexíveis no controle do potencial redox celular, existindo evidências de que tanto na condição de menor ou maior quantidade tecidual de mitocôndrias, oscilaçóes ocorridas entre os extremos de sua funcionalidade resultam em modificaçôes nas suas eficiências bioenergéticas. 


\section{Abstract \\ Metabolic ceiling as indicative of endurance capacity: importance of reserve oxidative capacity}

Compared with other animal species, the endurance capacity of humans is remarkable. The thesis of limiting and determining factors of endurance consider variables such as maximum oxygen $\left(\mathrm{O}_{2}\right)$ consumption $\left(\mathrm{VO}_{2 \max }\right)$, metabolic thresholds and movement economy the most importants. As the $\mathrm{O}_{2}$ and metabolic products of substrates (glucose and fatty acids) are used in muscle's mitochondria to obtain energy by oxidative processes during long duration exercise, this is another important variable to be considered. The aim of this assay is to show that the incidence of fatigue in this kind of exercise might be related to negative changes in mitochondrial oxidative potential. Mitochondrial oxidative potential is situated between the extreme limits of its functionality, which correspond to the states 4 (rest) and $3\left(\mathrm{VO}_{2 \text { max }}\right)$. The thesis put forward here is that when this variable is close to the state 3 during prolonged intense exercise, the body depletes their possibilities to produce energy through oxidative phosphorilation, with consequent changes in metabolic thresholds and increase in the percent of $\mathrm{VO}_{2 \max }$ used in this condition. Therefore, mitochondria function as a cellular bioenergetic thermostat during situations such as those involving prolonged strenuous exercise.

KEY words: Fatigue; Metabolism; Mitochondria; Endurance.

\section{Referências}

1. Sansbury BE, Jones SP, Riggs DW, Darley-Usmar VM, Hill BG. Bioenergetic function in cardiovascular cells: the importance of the reserve capacity and its biological regulation. Chem Biol Interact. 2011;191:288-95.

2. Jacobs RA, Rasmussen P, Siebenmann C, et al. Determinants of time trial performance and maximal incremental exercise in highly trained endurance athletes. J Appl Physiol. 2011;111:1422-30.

3. Bishop DJ, Granata C, Eynon N. Can we optimise the exercise training prescription to maximise improvements in mitochondria function and content? Biochim Biophys Acta. 2014;1840:1266-75.

4. Russell AP, Foletta VC, Snow RJ, Wadley GD. Skeletal muscle mitochondria: a major player in exercise, health and disease. Biochim Biophys Acta. 2014;1840:1276-84.

5. Tonkonogi M, Sahlin K. Physical exercise and mitochondrial function in human skeletal muscle. Exerc Sport Sci Rev. 2002;30:129-37.

6. Piersma T. Why marathon migrants get away with high metabolic ceilings: towards an ecology of physiological restraint. The J Exp Biol. 2011;214:295-302.

7. Krustrup P, Ferguson RA, Kjaer M, Bangsbo J. ATP and heat production in human skeletal muscle during dynamic exercise: higher efficiency of anaerobic than aerobic ATP resynthesis. J Physiol. 2003;549:255-69.

8. Brickley G, Doust J, Williams CA. Physiological responses during exercise to exhaustion at critical power. Eur J Appl Physiol. 2002;88:146-51.

9. Bishop CM. The maximum oxygen consumption and aerobic scope of birds and mammals: getting to the heart of the matter. Proc Biol Sci. 1999;266:2275-81.

10. Priede IG. Natural selection for energetic efficiency and the relationship between activity level and mortality. Nature. 1977;267:610-1.

11. Markovic G, Vucetic V, Nevill AM. Scaling behaviour of $\mathrm{VO}_{2}$ in athletes and untrained individuals. Ann Human Biol. 2007;34:315-28.

12. Saunders PU, Pyne DB, Telford RD, Hawley JA. Factors affecting running economy in trained distance runners. Sports Med. 2004;34:465-85.

13. Sokolova IM. Energy-limited tolerance to stress as a conceptual framework to integrate the effects of multiple stressors. Integr Comp Biol. 2013:1-12.

14. Layec G, Haseler LJ, Hoff J, et al. Short-term training alters the control of mitochondrial respiration rate before maximal oxidative ATP synthesis. Acta Physiol (Oxf). 2013;208:376-86. 
15. Desler C, Hansen TL, Frederiksen JB, Marcker ML, Singh KK, Juel Rasmussen L. Is there a link between mitochondrial reserve respiratory capacity and aging? J Aging Res. 2012;2012:192503.

16. Johnson ML, Robinson MM, Nair KS. Skeletal muscle aging and the mitochondrion. Trends Endocrinol Metab. 2013;24:247-56.

17. Conley KE, Jubrias SA, Cress ME, Esselman P. Exercise efficiency is reduced by mitochondrial uncoupling in the elderly. Exp Physiol. 2013;98:768-77.

18. Boullosa DA, Nakamura FY. The evolutionary significance of fatigue. Front Physiol. 2013;4:309.

19. Liesa M, Shirihai OS. Mitochondrial dynamics in the regulation of nutrient utilization and energy expenditure. Cell Metab. 2013;17:491-506.

20. Befroy DE, Petersen KF, Dufour S, Mason GF, Rothman DL, Shulman GI. Increased substrate oxidation and mitochondrial uncoupling in skeletal muscle of endurance-trained individuals. Proc Natl Acad Sci U S A. 2008;105:16701-6.

21. Kadenbach B. Intrinsic and extrinsic uncoupling of oxidative phosphorylation. Biochim Biophys Acta. 2003;1604:77-94.

22. Conley KE, Amara CE, Bajpeyi S, et al. Higher mitochondrial respiration and uncoupling with reduced electron transport chain content in vivo in muscle of sedentary versus active subjects. J Clin Endocrinol Metab. 2013;98:129-36.

23. Sawka MM, Leon LR, Montain SJ, Sonna LA. Integrated physiological mechanisms of exercise performance, adaptation, and maladaptation to heat stress. Compr Physiol. 2011;1:1883-928.

24. Aledo JC. Metabolic pathways: does the actual Gibbs free-energy change affect the flux rate? Biochem Mol Biol Educ. 2001;29:142-3.

25. Constable SH, Favier RJ, McLane JA, Fell RD, Chen M, Holloszy JO. Energy metabolism in contracting rat skeletal muscle: adaptation to exercise training. Am J Physiol. 1987;253:C316-22.

26. Larsen FJ, Schiffer TA, Borniquel S, et al. Dietary inorganic nitrate improves mitochondrial efficiency in humans. Cell Metab. 2011;13:149-59.

27. Larsen FJ, Schiffer TA, Sahlin K, Ekblom B, Weitzberg E, Lundberg JO. Mitochondrial oxygen affinity predicts basal metabolic rate in humans. FASEB J. 2011;25:2843-52.

28. Jobson SA, Hopker JG, Korff T, Passfield L. Gross efficiency and cycling performance: a brief review. J Sci Cycling. 2012;1:3-8

29. Schlagowski AI, Singh F, Charles AL, et al. Mitochondrial uncoupling reduces exercise capacity despite several skeletal muscle metabolic adaptations. J Appl Physiol. 2014;116:364-75.

\section{Agradecimentos}

O autor agradece aos Professores Doutores Edison de Jesus Manoel (Departamento de Pedagogia) e Rômulo Bertuzzi (Departamento de Esporte), ambos docentes da Escola de Educação Física e Esporte da Universidade de São Paulo (USP), pela leitura e valiosas sugestóes.

ENDEREÇO

Benedito Pereira

Departamento de Esporte

Escola de Educação Física e Esporte - USP

Av. Prof. Mello Moraes, 65

05508-030 - São Paulo - SP - BRASIL
Recebido para publicação: 30/09/2014

Aceito: 07/10/2014 